\section{Zervixkarzinom: schlechte Prognose bei hohen Monozyten-Werten}

\begin{abstract}
Periphere Monozyten spielen eine wichtige Rolle bei der Immunantwort gegen bestimmte Tumorerkrankungen. Als bisher prognostisch relevanter Tumormarker beim Zervixkarzinom gilt das SSC-Antigen. Wie jetzt gezeigt wurde, erlaubt die Kombination von Monozyten- und SCC-Wert vor Behandlungsbeginn eine bessere Vorhersage der Prognose beim Zervixkarzinom.
\end{abstract}

W eltweit ist das Zervixkarzinom die zweithäufigste maligne Neoplasie. Etwa $80 \%$ der invasiven Zervixkarzinome sind Plattenepithelkarzinome. Squamouscell-carcinoma (SSC)-Antigen (syn. Tumor-Antigen (TA)-4) gilt als spezifischer Tumormarker für zervikale Plattenepithelkarzinome. Es wurde gezeigt, dass die Konzentration von SSC-Antigen mit der neoplastischen Transformation des squamösen Zervixepithels ansteigt. Nach einer Behandlung weisen zunehmende SSCAntigenspiegel in 46-92\% der Fälle noch vor der klinischen Diagnose auf ein Rezidiv hin. Ungeklärt ist die Bedeutung von SSC-Antigen als prognostischer Marker vor einer Behandlung des Zervixkarzinoms. Antigen-präsentierende Zellen
(APC) wie Makrophagen und dendritische Zellen spielen eine wichtige Rolle für die Erkennung von Antigenen durch T-Lymphozyten. Neue Befunde zeigten, dass APCs, die von peripheren Monozyten abstammen, eine wesentliche Rolle bei der Initiierung, Programmierung und Regulation von tumorspezifischen Immunreaktionen spielen.

Nun wurde erstmals demonstriert, dass die Konzentration von zirkulierenden Monozyten mit dem Nachweis von Plattenepithelkarzinomen im HNOBereich sowie der Mortalität in Zusammenhang stehen. In dieser Studie aus Korea wurde die prognostische Bedeutung von zirkulierenden Monozyten im Vergleich zum SCC-Spiegel vor einer

\title{
Schützt Zöliakie vor gynäkologischen Tumoren?
}

- Schwedische Ärzte werteten die Daten von 17.852 Frauen aus, bei denen in den Jahren 1969 bis 2007 die Diagnose Zöliakie gestellt wurde, und berechneten mithilfe der Cox-Regression deren Risiko an einem hormonabhängigen Karzinom wie Brust-, Endometrium- und Ovarialkarzinom zu erkranken. Das Tumorrisiko stellten sie dem von 88.400 altersentsprechenden weiblichen Kontrollpersonen gegenüber.

Die Zöliakie-Patientinnen schnitten bei allen drei Tumorentitäten besser ab als die Kontrollgruppe (Brustkrebs: $\mathrm{HR}=0,85 ; 95 \% \mathrm{Cl}=0,72-1,01$; Endometriumkarzinom: $\mathrm{HR}$ $=0,60 ; 95 \% \mathrm{Cl}=0,41-0,86$; Ovarialkarzinom: $\mathrm{HR}=0,89 ; 95 \% \mathrm{Cl} 0,59-1,34)$. Beim Mammakarzinom etwa betrug das absolute Risiko für Frauen mit Dünndarmerkrankung 90/100.000 Personenjahre und lag damit deutlich niedriger als die 106/100.000 Personenjahre bei der Kontrollgruppe. Dabei war es unerheblich, ob die Diagnose der Zöliakie bereits vor oder erst nach der Menopause gestellt worden war.

Den protektiven Effekt dieser chronischen Darmerkrankung führen die Autoren auf die damit verbundene schlechtere Nahrungsverwertung zurück. Zöliakie-Patientinnen haben aufgrund einer Malabsorption einen geringeren BMI, was wiederum auch die reproduktive Phase beeinflusse, die Menarche trete später ein, die Menopause früher und die Ovarfunktion sei gestört. Alles in allem sei die Zeitspanne des Östrogeneinflusses verkürzt.

Dr. Dagmar Kraus

Ludvigsson JF et al. Reduced risk of breast, endometrial and ovarian cancer in women with celiac disease. Int J Cancer 2011; DOI: 10.1002/ijc.26454

Behandlung bei Patientinnen mit zervikalem Plattenepithelkarzinom untersucht. Retrospektiv wurden 788 Patientinnen aus den Jahren 1996-2007 mit Zervixkarzinomen (FIGO-Stadium IBIVA) in diese Studie eingeschlossen. Von allen Frauen lag ein Differenzialblutbild sowie der SSC-Wert vor, der mindestens zwei Wochen vor Behandlungsbeginn bestimmt wurde. Die Patientinnen wurden durchschnittlich 53,4 Monate nachbeobachtet und die Fünf-Jahres-Überlebenszeit lag bei $87,8 \%$. Die Konzentration der zirkulierenden Monozyten vor Behandlungsbeginn betrug $349 / \mu \mathrm{l}$ (21-1463), der SCC-Wert 1,6 ng/ml (0,1-362,0). Die multivariable Analyse ergab, dass die Monozyten-Konzentration ein unabhängiger prognostischer Faktor für ein progressionsfreies Überleben $(\mathrm{p}=0,007)$ ist sowie insgesamt auf eine bessere Prognose bei einer fortgeschrittenen lokalen Erkrankungen $(p=0,038)$ hinweist. Die SCC-Konzentration als Prognose-Instrument erlangte hingegen keine Signifikanz $(p=0,151)$ hinsichtlich der gesamten Überlebensrate bei Patientinnen mit lokal fortgeschrittenem Zervixkarzinom. Erhöhte Monozyten-Werte vor Behandlungsbeginn weisen auf eine schlechtere Prognose hin. Die Autoren folgern aus den Ergebnissen der Studie, dass eine Kombination der Monozyten- und der SSC-Werte die prognostische Aussage des alleinigen SSCWertes bei Patientinnen mit lokalem fortgeschrittenen zervikalen Plattenepithelkarzinom verbessern würde.

Fazit: Nicht zuletzt seit dem letztjährigen Nobelpreis für Medizin ist die Bedeutung von dendritischen Zellen gestiegen. Monozyten, die von diesen Zellen abstammen, sind auch in der Ätiologie von Tumoren, wie hier am Zervixkarzinom gezeigt, von möglicher Relevanz. Die Bestimmung von Monozyten im peripheren Blut, eine einfache und kostengünstige Laboruntersuchung, könten sich jetzt als neuer Tumormarker beim Zervixkarzinom etablieren.

Prof. Tino F. Schwarz

Lee YY et al: Prognostic value of pre-treatment circulatig monocyte count in patients with cervical cancer: comparison with SCCAg levels. Gynecol Oncol 2012; 124: 92-97 